\title{
Clinicopathological Characteristics and Recurrence-Free Survival of Rare Variants of Papillary Thyroid Carcinomas in Korea: A Retrospective Study
}

\author{
Mijin Kim ${ }^{1, *}$, Sun Wook Cho ${ }^{2 *}$, Young Joo Park ${ }^{2}$, Hwa Young $\mathrm{Ahn}^{3}$, Hee Sung Kim ${ }^{4}$, Yong Joon Suh ${ }^{5}$, Dughyun Choi ${ }^{6}$, \\ Bu Kyung Kim ${ }^{7}$, Go Eun Yang ${ }^{8}$, Il-Seok Park ${ }^{9}$, Ka Hee Yi ${ }^{10}$, Chan Kwon Jung ${ }^{11}$, Bo Hyun Kim ${ }^{1}$ \\ ${ }^{1}$ Department of Internal Medicine, Biomedical Research Institute, Pusan National University Hospital, Busan; ${ }^{2}$ Department of \\ Internal Medicine, Seoul National University Hospital; ${ }^{3}$ Division of Endocrinology, Department of Internal Medicine, \\ ${ }^{4}$ Department of Pathology, Chung-Ang University College of Medicine, Seoul; ${ }^{5}$ Department of Breast and Endocrine Surgery, \\ Hallym University Sacred Heart Hospital, Anyang; ${ }^{6}$ Division of Endocrinology and Metabolism, Department of Internal \\ Medicine, Soonchunhyang University Bucheon Hospital, Soonchunhyang University College of Medicine, Bucheon; \\ ${ }^{7}$ Department of Internal Medicine, Kosin University College of Medicine, Busan; ${ }^{8}$ Department of Radiology, School of \\ Medicine, Kangwon National University Hospital, Chuncheon; ${ }^{9}$ Department of Otorhinolaryngology-Head and Neck Surgery, \\ Hallym University Dongtan Sacred Heart Hospital, Hallym University College of Medicine, Hwaseong; ${ }^{10}$ Department of \\ Internal Medicine, Seoul Metropolitan Government Seoul National University Boramae Medical Center; ${ }^{11}$ Department of \\ Hospital Pathology, College of Medicine, The Catholic University of Korea, Seoul, Korea
}

Background: We aimed to evaluate the clinicopathological features and biological behaviors of Korean thyroid cancer patients with rare variants of papillary thyroid carcinoma (PTC) to address the ambiguity regarding the prognostic consequences of these variants. Methods: We retrospectively reviewed the medical records of 5,496 patients who underwent thyroid surgery for PTC, between January and December 2012, in nine tertiary hospitals. Rare PTC variants included tall cell (TCV), columnar cell (CCV), diffuse sclerosing (DSV), cribriform-morular (CMV), solid (SV), hobnail, and Warthin-like variants. Recurrence-free survival (RFS) was defined as the time from the date of thyroidectomy until recurrence.

Results: Rare variants accounted for $1.1 \%(n=63)$ of the PTC patients; with $0.9 \% \mathrm{TCV}, 0.02 \% \mathrm{CCV}, 0.1 \% \mathrm{DSV}, 0.1 \% \mathrm{CMV}$, and $0.1 \% \mathrm{SV}$. The mean age of patients and primary tumor size were $42.1 \pm 13.1$ years and $1.3 \pm 0.9 \mathrm{~cm}$, respectively. Extrathyroidal extension and cervical lymph node metastasis were observed in 38 (60.3\%) and $37(58.7 \%)$ patients, respectively. Ultrasonographic findings revealed typical malignant features in most cases. During a median follow-up of 7 years, $6.3 \%$ of patients experienced a locoregional recurrence. The 5-year RFS rates were $71.4 \%$ in patients with DSV or SV, $95.9 \%$ for TCV, or CCV, and $100 \%$ for other variants. DSV emerged an independent risk factor associated with shorter RFS.

Received: 28 January 2021, Revised: 19 April 2021, Accepted: 4 May 2021

Corresponding authors: Bo Hyun Kim

Department of Internal Medicine, Biomedical Research Institute, Pusan National University Hospital, 305 Gudeok-ro, Seo-gu, Busan 49241, Korea

Tel: +82-51-240-7236, Fax: +82-51-254-3237, E-mail: pons71@hanmail.net

Chan Kwon Jung

Department of Pathology, College of Medicine, The Catholic University of Korea, 222 Banpo-daero, Seocho-gu, Seoul 06591, Korea

Tel: + 82-2-2258-1622, Fax:+82-2-2258-1627, E-mail: ckjung@catholic.ac.kr

\section{Copyright $(92021$ Korean Endocrine Society}

This is an Open Access article distributed under the terms of the Creative Commons Attribution Non-Commercial License (https://creativecommons.org/ licenses/by-nc/4.0/) which permits unrestricted non-commercial use, distribution, and reproduction in any medium, provided the original work is properly cited.

*These authors contributed equally to this work. 
Conclusion: In this multicenter Korean cohort, rare variants accounted for $1.1 \%$ of all PTC cases, with TCV being the most frequent subtype. DSV emerged as a significant prognostic factor for RFS.

Keywords: Thyroid neoplasms; Carcinoma, papillary; Prognosis; Recurrence; Prevalence

\section{INTRODUCTION}

Papillary thyroid carcinoma (PTC) is the most common thyroid malignancy with an indolent clinical course [1,2]. Classical PTC accounts for $55 \%$ to $65 \%$ of the cases; while, more than 10 different variants have been identified [3]. Among these, follicular variant is a common subtype, accounting for about $15 \%$ to $30 \%$ of all PTCs, and its incidence has increased in recent times [4-7]. The other, relatively rare subtypes include tall cell (TCV), columnar cell (CCV), diffuse sclerosing (DSV), cribriformmorular (CMV), solid (SV), hobnail (HV), and Warthin-like variants $[8,9]$. The prevalence of these variants differs between regions, countries, and patient populations [9]. The incidence of TCV ranges from $4 \%$ to $16 \%$, and it does not constitute a common pathological entity when a strict pathologic definition is applied $[5,7,10,11]$. The other variants are rarer, accounting for $1 \%$ to $3 \%$ of PTC [10-21]. Owing to their rarity, most studies on PTC variants largely consist of case reports with few large-sample analyses or multicenter cross-sectional studies [9].

Rare variants have distinct clinical, pathological, and prognostic characteristics [8]. Certain variants have more aggressive or, conversely, more favorable tumor behaviors than classic PTCs; therefore, they contribute to risk stratification [8]. According to the American Thyroid Association (ATA) initial risk stratification, PTCs are classified into three major groups based on their biological behaviors: (1) aggressive variants with unfavorable outcomes, including TCV, CCV, and $\mathrm{HV}$; (2) variants with less favorable outcomes but controversy exists regarding their mortality rate, including SV and DSV; and (3) other variants [8]. However, the prognostic implications of these PTC variants remain controversial [8]. For example, a Korean singlecentered study, conducted on 144 patients with aggressive variants of PTC, revealed that patients with TCV did not exhibit worse clinical outcomes when compared to those with classic PTC, diagnosed with similar clinicopathological factors [10]. $\mathrm{CCV}$ was associated with poorer clinical outcomes after propensity score matching [10]. Additionally, some studies suggest that SV and DSV could be classified as a high-risk pathological entity due to their propensity for recurrence [14,15,22-26]. A large, multicentered study in France revealed that patients with
DSV were at a nine times higher risk of recurrence than patients with other variants of PTC, despite aggressive surgical interventions that required more frequent follow-ups [15]. Nevertheless, these variants usually occur in young patients and are highly responsive to treatment, with a lower mortality rate. Therefore, there is a lack of clarity regarding the pathological subtypes that require more aggressive treatment and careful surveillance.

In this multicentered study conducted in Korea, we aimed to evaluate the clinicopathologic characteristics and biological behaviors of patients with rare variants of PTC using a large, multicenter cohort of thyroid cancer patients. Additionally, we elucidated the prognostic factors associated with recurrence-free survival (RFS) in PTC patients with rare variants.

\section{METHODS}

\section{Patients}

We retrospectively reviewed the medical records of 5,496 consecutive patients who underwent thyroid surgery for PTC, between January 2012 and December 2012, at nine tertiary hospitals in the Republic of Korea. All patients were above 18 years of age. Of these, we identified 63 patients with uncommon pathological variants; including cases other than classical or follicular variant PTC. The largest tumor was considered as the primary lesion in cases of multiple tumor foci with different sizes. From the electronic medical records, we obtained their clinical and pathological data, including age, sex, primary tumor size, ultrasonography (US) findings, pathological report, extent of surgery, presence of distant metastases, use of radioactive iodine (RAI) ablation, RAI dose, recurrence, date of recurrence, survival status, date of last follow-up, and cause of death. Recurrence was defined as the appearance of pathologically confirmed malignant tissue and/or metastatic lesions. RFS was defined as the time from the date of thyroidectomy until recurrence.

The relevant Institutional Review Boards approved our study protocol (Pusan National University Hospital, 1906-002-079; Seoul National University Hospital, 1905-155-1035; ChungAng University Hospital, 1906-006-16266; Hallym University Dongtan Sacred Heart Hospital, 2019-06-011; Soonchunhyang University Bucheon Hospital, 2019-05-014; Hallym University 
Sacred Heart Hospital, 2020-09-015; Kosin University Gospel Hospital, 2019-05-015; Kangwon National University Hospital, KNUH-2019-06-002; Seoul St. Mary's Hospital, KC16SISI0104). They waived the requirement of informed consent from the participants due to the retrospective design of the study.

\section{Classification of histologic variants}

Practicing pathologists made pathologic diagnosis and classified the histologic variants of PTCs in their routine clinical practice, using the 2004 World Health Organization classification of tumors of endocrine organs [27]. According to this classification, PTC was diagnosed as TCV when tall cells with a height-to-width ratio of $\geq 3$ comprised more than $50 \%$ of all tumor cells [27].

\section{Variant groups}

Rare variants of PTC included TCV, CCV, DSV, CMV, SV, HV, and Warthin-like variants. These variants were categorized into the following three groups based on the ATA guidelines [8]: (1) aggressive variants, including TCV, CCV, and HV; (2) those (SV and DSV) with less favorable outcomes but controversy exists regarding mortality rate; and (3) other uncommon variants, including CMV and Warthin-like variant.

\section{Ultrasonography analysis}

Experienced radiologists from each institution investigated the US features of the tumors, using a picture archiving and communication system. These features included the composition, echogenicity, calcification, margin, and shape of the tumors. For every nodule, we used the consensus ratings for each US feature to classify the risk of malignancy, according to the 2015 guidelines of the ATA classification [8]. The information on preoperative US images of three patients was unavailable.

\section{Statistical analysis}

Statistical analyses were performed using the R program version 3.6.2 (The R Project for Statistical Computing, Vienna, Austria). Survival curves and forest plot were plotted using GraphPad Prism version 5.0 (GraphPad Software Inc., San Diego, CA, USA) and MedCalc Statistical Software version 19.5.3 (MedCalc Software Ltd., Ostend, Belgium), respectively. Continuous variables are presented as mean \pm standard deviation, and categorical variables are presented as numbers (\%). RFS curves were plotted using the Kaplan-Meier method, and the log-rank test was used to determine the significance of difference. The relative risks for RFS were evaluated using the Cox proportional hazards model and presented as hazard ratios (HRs), 95\% confidence intervals (CIs), and $P$ values. The Cox proportional hazards model was also used to analyze the prognostic factors associated with RFS. All $P$ values were two-sided, and $P<0.05$ was considered statistically significant.

\section{RESULTS}

\section{Clinicopathological characteristics}

Rare variants of PTC were found in $63(1.1 \%)$ of 5,496 patients
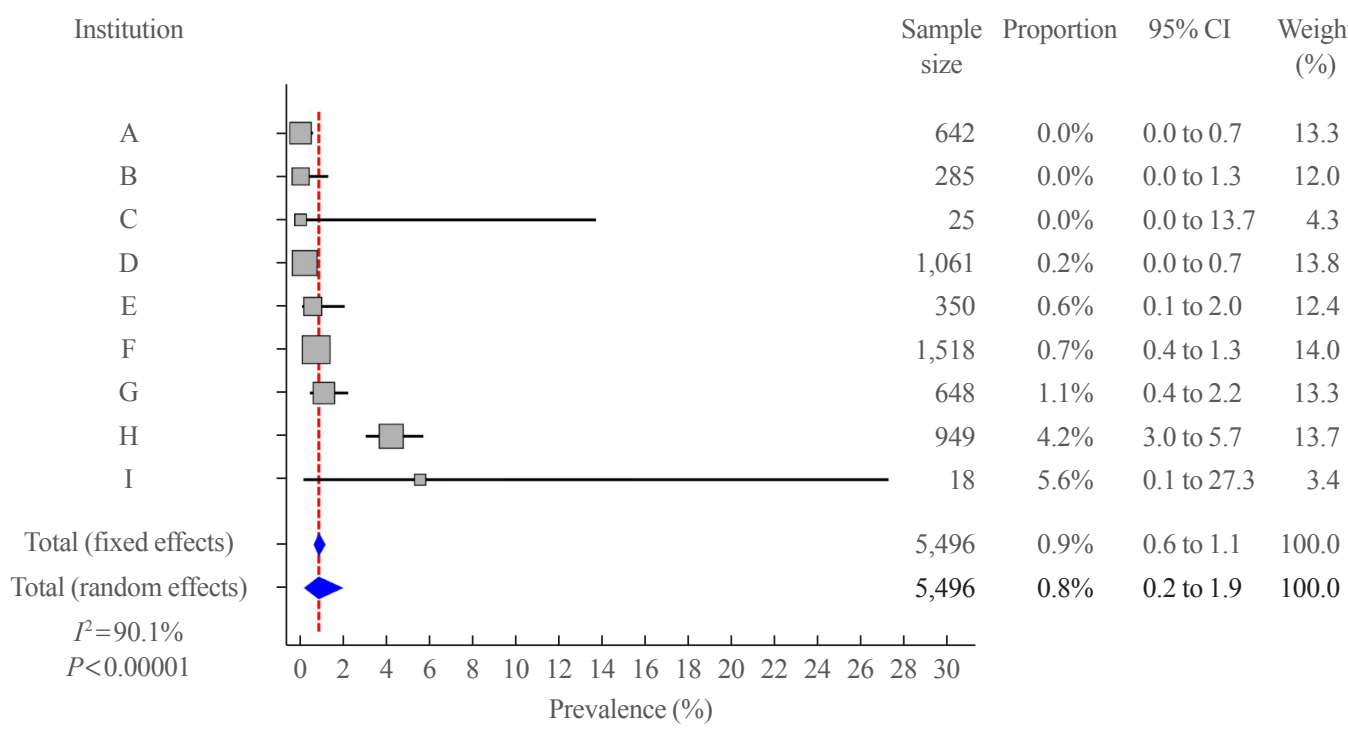

Fig. 1. Forest plot for the overall pooled prevalence of rare variants of papillary thyroid carcinoma. The size of the box represents the weight given to each institution. The whiskers indicate the $95 \%$ confidence interval (CI). 


\begin{tabular}{lc} 
Table 1. Baseline Characteristics of Patients \\
of Papillary Thyroid Carcinoma \\
\hline Characteristic & \\
\hline Number of patients & Value \\
Age, yr & 63 \\
Male patients & $42.1 \pm 13.1$ \\
Pathology, variant & $10(15.9)$ \\
Tall cell & $51(81.0)$ \\
Columnar cell & $1(1.6)$ \\
Diffuse sclerosing & $4(6.3)$ \\
Cribriform-morular & $3(4.8)$ \\
Solid & $4(6.3)$ \\
Hobnail & 0 \\
Warthin-like & 0 \\
Primary tumor size, cm & $1.3 \pm 0.9$ \\
Lymphovascular invasion & $22(34.9)$ \\
Extrathyroidal extension & \\
Microscopic & $304.4 \pm 74.8$ \\
Gross & $30(47.6)$ \\
Cervical LN metastasis & $8(12.7)$ \\
N1a & \\
N1b & $29(46.0)$ \\
Distant metastasis & $8(12.7)$ \\
Total thyroidectomy & 0 \\
RAI & $59(93.7)$ \\
\hline
\end{tabular}

Values are expressed as mean \pm standard deviation or number (\%). LN, lymph node; RAI, radioiodine ablation.

with PTC and included $51(0.9 \%) \mathrm{TCV}$, one $(0.02 \%) \mathrm{CCV}$, four $(0.1 \%) \mathrm{DSV}$, three $(0.1 \%) \mathrm{CMV}$, and four $(0.1 \%) \mathrm{SV}$ cases. HV and Warthin-like variants were not identified in this cohort. The incidence of rare variants varied from $0 \%$ to $5.6 \%$ of all PTCs from nine institutions. The overall pooled prevalence of rare variants was $0.8 \%$ (95\% CI, 0.2 to 1.9 ) as shown in Fig. 1.

Table 1 lists the baseline characteristics of the 63 PTC patients with rare variants. The mean age of patients was $42.1 \pm$ 13.1 years, and $15.9 \%(n=10)$ of them were males. The mean primary tumor size was $1.3 \pm 0.9 \mathrm{~cm}$, and $33(52.4 \%)$ patients had tumors larger than $1 \mathrm{~cm}$. Lymphovascular invasion, microscopic extrathyroidal extension (ETE), and gross ETE were observed in $22(34.9 \%), 30(47.6 \%)$ and eight (12.7\%) patients, respectively. Cervical lymph node (LN) metastases were detected in $37(58.7 \%)$ patients, including 29 (46.0\%) patients with N1a stage and eight (12.7\%) patients with N1b stage metasta-
Table 2. Ultrasonographic Features

\begin{tabular}{lc}
\hline Variable & Number (\%) \\
\hline ATA classification & $45(75.0)$ \\
High suspicion & $15(25.0)$ \\
Intermediate suspicion & 0 \\
Low suspicion & 0 \\
Very low suspicion & 0 \\
Benign & \\
Echogenicity & $50(83.3)$ \\
Hypoechoic & $10(16.7)$ \\
Isoechoic & \\
Calcification & $26(43.3)$ \\
Microcalcification & $4(6.7)$ \\
Macrocalcification & 0 \\
Rim calcification & $2(3.3)$ \\
Micro- and macrocalcification & $46(76.7)$ \\
Margin, irregular or microlobulated & $22(36.7)$ \\
Shape, taller-than-wide & \\
\hline ATA, American Thyroid Association. &
\end{tabular}

ses. None of the patients were diagnosed with distant metastases. Most patients underwent total thyroidectomy (93.7\%) and 10 patients initially underwent lateral neck dissection. All patients who underwent hemithyroidectomy had a tumor sized $<1$ $\mathrm{cm}$, and no patients underwent completion thyroidectomy. Fiftythree patients $(84.1 \%)$ received RAI therapy with a mean dose of $104.4 \pm 74.8 \mathrm{mCi}$. Based on the ATA classification, we classified $45(75 \%)$ and $15(25 \%)$ patients into high and intermediate suspicion categories, respectively (Table 2). The prominent US features of these tumors included hypoechogenicity $(83.3 \%)$, microcalcification (43.3\%), irregular or microlobulated margins (76.7\%), and taller-than-wide shape (36.7\%).

\section{Recurrence-free survival}

During the median follow-up of 7 years, locoregional recurrences occurred in four patients: two patients each with TCV and DSV. These patients underwent total thyroidectomy with neck LN dissection (central only for three patients, and central plus lateral for one patient) initially, and three of them had an N1a tumor. All recurrences were confirmed pathologically; one was initially detected using a post-RAI scan, and the others using follow-up US images. The 5-year RFS rate for rare variants in PTC patients was 93.3\%, and the RFS curves significantly differed according to variant subtypes $(P=0.004)$ (Fig. 2A). Ad- 

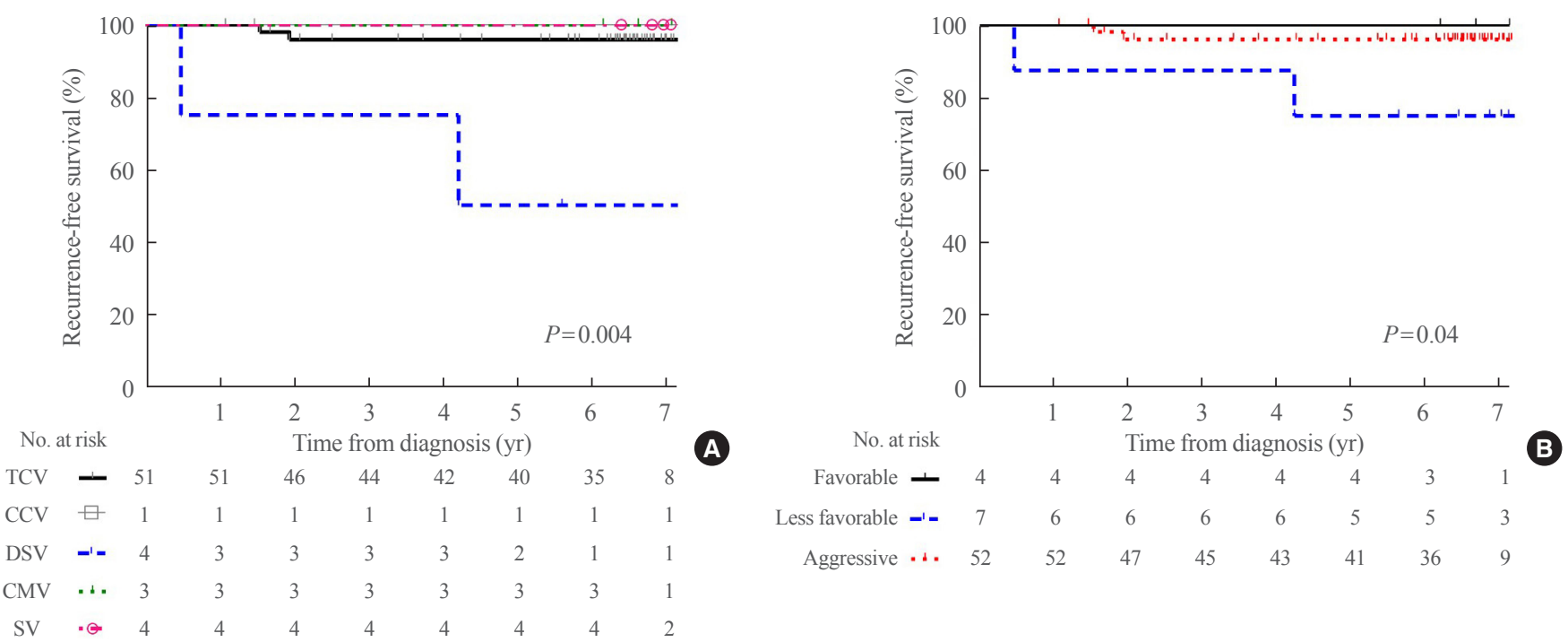

Fig. 2. (A) Recurrence-free survival curves according to each pathological variant. (B) Recurrence-free survival curves according to the American Thyroid Association classification: variants with aggressive outcomes include tall cell (TCV), columnar cell (CCV), and hobnail variants; those with less favorable outcomes include solid (SV) and diffuse sclerosing variants (DSV); and those with favorable outcomes include cribriform-morular (CMV), and Warthin-like variants.

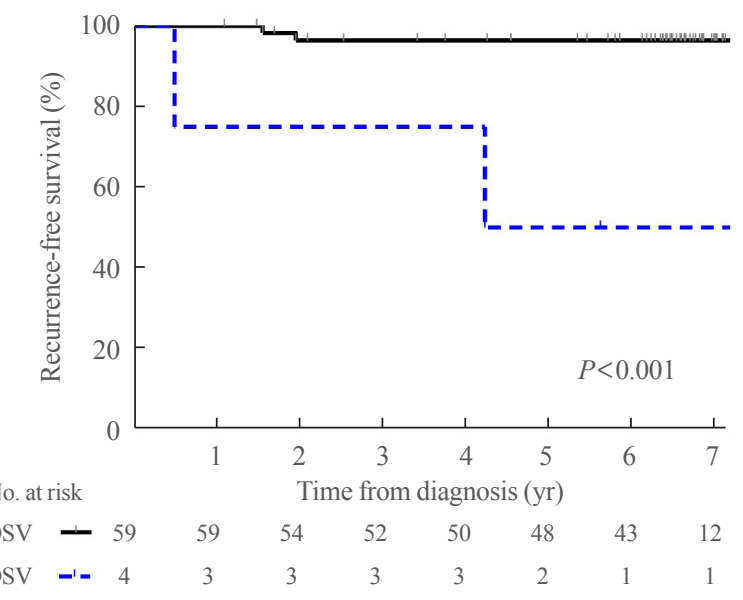

Fig. 3. Recurrence-free survival curves of patients with diffuse sclerosing variant (DSV) and others.

ditionally, we evaluated RFS according to the clinical behaviors of the variants; the 5-year RFS rate for variants with favorable, less favorable, and aggressive outcomes were $100.0 \%, 71.4 \%$, and $95.9 \%$, respectively (Fig. 2B). Interestingly, patients with less favorable outcomes had worse RFS than those with aggressive outcomes (HR, 7.7; 95\% CI, 1.1 to $54.8 ; P=0.041$ ). Additionally, we performed a subgroup analysis to identify the prognostic factors in patients with rare variants. Patients with DSV had significantly poorer RFS than those with other variants (HR, 17.1; 95\% CI, 2.4 to 121.7; $P=0.005$ ) (Fig. 3). There was no significant difference in RFS with respect to other factors, such as age, sex, primary tumor size, lymphovascular invasion, ETE, RAI therapy, and US features (data not shown).

\section{Characteristics of PTC patients with DSV}

Table 3 summarizes the data on clinical and pathological characteristics of the four patients with DSV. All patients were between 20 and 30 years of age, and three (75\%) were females. Three of the patients had primary tumors larger than $1 \mathrm{~cm}$. All patients with DSV underwent total thyroidectomy and developed neck LN metastases with extensive LN involvement (range, 2 to 29). A 33-year-old male underwent total thyroidectomy and neck dissection, with pathology reporting 29 of $61 \mathrm{LN}$ positive for DSV subtype of PTC. Preoperative US and computed tomography images showed no evidence of suspicious LNs in both sides of the neck. The 6-month post-RAI scan showed activity in the right level III LN. A right lateral neck dissection was performed, and pathology reported one of $7 \mathrm{LN}$ to be positive for PTC. Three patients (75\%) had pathological features of Hashimoto's thyroiditis. The US findings indicated that the DSV tumors could be classified into high suspicion $(50 \%)$ or intermediate suspicion (50\%) categories; however, these features are not helpful in predicting recurrence at the time of diagnosis. Two patients $(50 \%)$ developed disease recurrence in the lateral neck at 6 and 51 months after thyroidectomy, respectively. 
Table 3. Clinical Characteristics of the Four Papillary Thyroid Carcinoma Patients with Diffuse Sclerosing Variant

\begin{tabular}{|c|c|c|c|c|c|c|c|c|c|c|}
\hline Patient & Age, yr & Sex & $\begin{array}{c}\text { Primary } \\
\text { tumor size, } \\
\mathrm{cm}\end{array}$ & TNM stage $^{\mathrm{a}}$ & $\begin{array}{c}\text { Metastatic } \\
\text { LNs }\end{array}$ & Background & US category $^{\mathrm{b}}$ & Recur & Recurred area & RFS, mo \\
\hline 1 & 33 & M & 3.5 & T3N1aM0 & 29 & None & Intermediate & Yes & Lat. neck LNs & 6 \\
\hline 2 & 21 & $\mathrm{~F}$ & 1.1 & T1N1aM0 & 6 & HT & Intermediate & Yes & Lat. neck LNs & 51 \\
\hline 3 & 32 & $\mathrm{~F}$ & 2.0 & T3N1bM0 & 22 & HT & High & No & - & 88 \\
\hline 4 & 26 & $\mathrm{~F}$ & 0.9 & T1N1aM0 & 2 & HT & High & No & - & 67 \\
\hline
\end{tabular}

TNM, tumor-node-metastasis; LN, lymph node; US, ultrasonography; RFS, recurrence-free survival; HT, Hashimoto's thyroiditis; Lat., lateral.

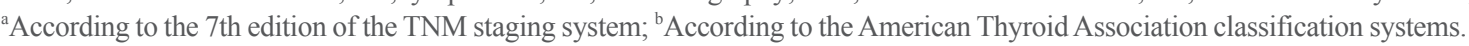

\section{DISCUSSION}

Our retrospective investigation is the first large, multicenter study in Korea that evaluated the clinicopathological characteristics and RFS of the rare variants of PTC. Out of the 5,496 patients included in the study, only 63 patients exhibited rare variants of PTC, accounting for $1.1 \%$ of all PTC cases. TCV was the most frequent variant with an incidence of $0.9 \%$. We determined that some PTC patients with rare variants exhibited aggressive features; $12.7 \%$ each demonstrated gross ETE and lateral neck LN metastasis. Based on the ATA classification and US features, we classified all tumors into high or intermediate suspicion categories, with most belonging to the former category. During the follow-up period, locoregional recurrence occurred in $6.3 \%$ of the patients. The 5 -year RFS rates for variants with favorable, less favorable, and aggressive outcomes were $100 \%, 71.4 \%$, and $95.9 \%$, respectively. Additionally, DSV emerged as an independent risk factor associated with a shorter RFS.

The pathological definitions of these variants of PTC have changed over time, thus varying the incidences of these tumors accordingly $[9,27]$. We defined TCV as a tumor with more than $50 \%$ tall cells that are $\geq 3$ times as tall as wide. This rigorous definition may have contributed to a lower incidence of TCV compared to that previously reported $[5,7,10,11]$. A recent World Health Organization classification defined that tall cells should account for more than $30 \%$ of all tumor cells for the diagnosis of TCV. However, evidence shows that a tumor with more than $50 \%$ tall cells significantly and independently affects prognosis, whereas that with $30 \%$ to $49 \%$ tall cells does not [28]. Standardizing diagnostic criteria and accurately diagnosing rare variants of PTC are important to determine the patients who require extensive therapies, including a completion thyroidectomy followed by RAI ablation and aggressive surveil- lance.

This retrospective study was designed to examine the incidence and biological behaviors of rare variants of PTC, based on the pathology reports of patients with PTC. All pathology slides were reviewed by institutional pathologists to better represent clinical practice, and the incidence of rare variants varied from $0 \%$ to $5.6 \%$ for all PTCs in each institution. The uncommon variants may be easily overlooked by inexperienced pathologists, and these variants of PTC might be underdiagnosed by general pathologists, who tend to classify rare variants as classical PTC. This might be an explanation for the wide range of incidence of rare variants in this study and previous studies [29-31]. The incidence of TCV has been reported to range from $1.3 \%$ to $13 \%$ of all PTCs [29]. When carefully scrutinized for the diagnosis of TCV by endocrine pathologists, the proportion of TCV has been observed to increase [29]. The proportions of TCV diagnosed at a single institution in Korea were $4.9 \%$ and $6.8 \%$ of all PTCs in 2013 and in 2020, respectively [30,31], when all pathology slides were reviewed by an expert.

In the present study, most of the rare variants of PTC exhibited typical malignant features on US: solid hypoechoic nodules with irregular/microlobulated margins and microcalcifications. Little information exists on the imaging features of these rare variants of PTC due to their rareness [32,33]. In a retrospective study of eight patients with $10 \mathrm{TCVs}$, TCV appeared as markedly hypoechoic nodules with microlobulated margins, microcalcifications, and ETE [34]. SVs also were diagnosed as high or intermediate suspicion nodules on US [32,35]. Yun et al. [36], in their study of 17 DSV cases, described the characteristic US features that included diffuse and scattered microcalcifications with heterogeneous hypoechogenicity. On the contrary, CMVs that have indolent tumors did not exhibit malignant features on US [37]. Therefore, US features can be considered as indicators of tumor aggressiveness. Further research may help determine 
if US features can effectively predict prognosis in these variants of PTC. In this study, we found it difficult to assign specific characteristics for each variant and determine their prognostic implications due to a high proportion of patients with TCV and a wide overlap of US features among the variants.

Using ATA classification, the 5-year RFS rates for variants with favorable, less favorable, and aggressive outcomes were $100 \%, 71.4 \%$, and $95.9 \%$, respectively. In our study, the variants with less favorable outcomes had a significantly shorter RFS than those with aggressive outcomes. One possible explanation for our findings is the proportion of variants included in our analysis. None of our patients exhibited CCV and HV, which have been reported to be associated with recurrence, metastasis, and disease-specific death [10,38]. Another possibility is that we evaluated only for RFS, and not for disease-specific survival. Although DSV has more aggressive pathological features and are treated more aggressively than classical PTC, most studies revealed that it did not depict any adverse effects or a higher mortality rate $[13,14]$. However, a systematic review of DSV demonstrated that it was associated with worse overall survival [39]. Evaluating the long-term outcomes with a larger cohort may help explain the prognostic significance of each pathological variant.

Consistent with previous studies, our data indicated that DSV occurs at a younger age with a high incidence of cervical LN metastasis [22,24]. Additionally, a higher prevalence of underlying Hashimoto's thyroiditis and a higher female-to-male ratio are unique clinical features of DSV [13]. Abundant lymphocytic infiltration is a characteristic of DSV, and the molecular profile of DSV may be a possible explanation for its pathological difference from classical PTC [40]. RET/PTC rearrangements are common in DSV, whereas $B R A F$ mutations are observed in DSV at a lower frequency compared to classical PTC $[41,42]$. Our analysis of the Cox proportional hazards model revealed that DSV is a significant prognostic factor for RFS among patients with rare variants of PTC. It is noteworthy that all recurrences were locoregional and that the preoperative US features were not helpful in predicting recurrence. This suggests that close monitoring of the operation bed and lateral neck LNs is of importance during the follow-up after initial therapy for patients with DSV.

The present study has several limitations. First, this study is retrospective by design and involves a relatively small number of PTC patients with rare variants due to their inherent rareness and short period of data collection. This retrospective multicenter study was conducted after reviewing all pathology slides reported as PTC in each institution. We reviewed patients diagnosed with PTC over a year to allow more institutions to participate, and actually reviewed all pathology slides for 5,496 PTC cases. We also intend to evaluate the prognosis of patients with a follow-up period of 5 years or longer in patients diagnosed with a rare variant of PTC in 2012. Second, we failed to conduct centralized pathological examinations in this multicentered study, and there may have been discrepancies among the pathologists' interpretation of the diagnosis criteria. Third, the median follow-up duration of 7 years was relatively short to evaluate the recurrence. Additionally, no disease-specific death occurred during this period. Further studies using cohorts with longer data collection periods will yield more definitive results. However, this is the first multicenter cohort study to evaluate the clinicopathological characteristics and biological behaviors of rare variants of PTC in Korea.

In conclusion, our multicentered Korean cohort revealed that rare variants of PTC accounted for $1.1 \%$ of all PTCs with TCV being the most frequent variant. Locoregional recurrences occurred in four patients, and DSV was the only variant that emerged as a significant prognostic factor for RFS. These findings suggest that pathological variant should be taken into consideration while determining the follow-up and treatment strategies in patients with PTC. Further studies conducted on a larger scale are needed to verify the incidence, clinicopathological characteristics, and biologic behaviors.

\section{CONFLICTS OF INTEREST}

No potential conflict of interest relevant to this article was reported.

\section{ACKNOWLEDGMENTS}

This work was supported by the Korean Thyroid Association Clinical Research Award 2019.

\section{AUTHOR CONTRIBUTIONS}

Conception or design: Y.J.P., K.H.Y., C.K.J., B.H.K. Acquisition, analysis, or interpretation of data: M.K., S.W.C., H.Y.A., H.S.K., Y.J.S., D.C., B.K.K., G.E.Y., I.S.P., K.H.Y., C.K.J., B.H.K. Drafting the work or revising: M.K., S.W.C., C.K.J., B.H.K. Final approval of the manuscript: M.K., S.W.C., H.Y.A., H.S.K., Y.J.S., D.C., B.K.K., G.E.Y., I.S.P., K.H.Y., C.K.J., B.H.K. 


\section{ORCID}

Mijin Kim https://orcid.org/0000-0002-1538-8859

Sun Wook Cho https://orcid.org/0000-0002-7394-3830

Chan Kwon Jung https://orcid.org/0000-0001-6843-3708

Bo Hyun Kim https://orcid.org/0000-0001-9632-9457

\section{REFERENCES}

1. Hundahl SA, Fleming ID, Fremgen AM, Menck HR. A National Cancer Data Base report on 53,856 cases of thyroid carcinoma treated in the U.S., 1985-1995 [see comments]. Cancer 1998;83:2638-48.

2. Jonklaas J, Sarlis NJ, Litofsky D, Ain KB, Bigos ST, Brierley JD, et al. Outcomes of patients with differentiated thyroid carcinoma following initial therapy. Thyroid 2006;16: 1229-42.

3. Nikiforov YE, Biddinger PW, Thompson LDR. Diagnostic pathology and molecular genetics of the thyroid. 2nd ed. Philadelphia: Wolters Kluwer; 2013.

4. Nikiforov YE, Seethala RR, Tallini G, Baloch ZW, Basolo F, Thompson LD, et al. Nomenclature revision for encapsulated follicular variant of papillary thyroid carcinoma: a paradigm shift to reduce overtreatment of indolent tumors. JAMA Oncol 2016;2:1023-9.

5. Shi X, Liu R, Basolo F, Giannini R, Shen X, Teng D, et al. Differential clinicopathological risk and prognosis of major papillary thyroid cancer variants. J Clin Endocrinol Metab 2016;101:264-74.

6. Bychkov A, Hirokawa M, Jung CK, Liu Z, Zhu Y, Hong $\mathrm{SW}$, et al. Low rate of noninvasive follicular thyroid neoplasm with papillary-like nuclear features in Asian practice. Thyroid 2017;27:983-4.

7. Fagin JA, Wells SA Jr. Biologic and clinical perspectives on thyroid cancer. N Engl J Med 2016;375:1054-67.

8. Haugen BR, Alexander EK, Bible KC, Doherty GM, Mandel SJ, Nikiforov YE, et al. 2015 American Thyroid Association management guidelines for adult patients with thyroid nodules and differentiated thyroid cancer: the American Thyroid Association guidelines task force on thyroid nodules and differentiated thyroid cancer. Thyroid 2016;26:1133.

9. Kakudo K, Bychkov A, Bai Y, Li Y, Liu Z, Jung CK. The new 4th edition World Health Organization classification for thyroid tumors, Asian perspectives. Pathol Int 2018;68:64164.
10. Song E, Jeon MJ, Oh HS, Han M, Lee YM, Kim TY, et al. Do aggressive variants of papillary thyroid carcinoma have worse clinical outcome than classic papillary thyroid carcinoma? Eur J Endocrinol 2018;179:135-42.

11. Ito Y, Hirokawa M, Uruno T, Kihara M, Higashiyama T, Takamura Y, et al. Prevalence and biological behaviour of variants of papillary thyroid carcinoma: experience at a single institute. Pathology 2008;40:617-22.

12. Jiang C, Cheng T, Zheng X, Hong S, Liu S, Liu J, et al. Clinical behaviors of rare variants of papillary thyroid carcinoma are associated with survival: a population-level analysis. Cancer Manag Res 2018;10:465-72.

13. Chow SM, Chan JK, Law SC, Tang DL, Ho CM, Cheung WY, et al. Diffuse sclerosing variant of papillary thyroid carcinoma: clinical features and outcome. Eur J Surg Oncol 2003;29:446-9.

14. Akaishi J, Sugino K, Kameyama K, Masaki C, Matsuzu K, Suzuki A, et al. Clinicopathologic features and outcomes in patients with diffuse sclerosing variant of papillary thyroid carcinoma. World J Surg 2015;39:1728-35.

15. Chereau N, Giudicelli X, Pattou F, Lifante JC, Triponez F, Mirallie E, et al. Diffuse sclerosing variant of papillary thyroid carcinoma is associated with aggressive histopathological features and a poor outcome: results of a large multicentric study. J Clin Endocrinol Metab 2016;101:4603-10.

16. Sywak M, Pasieka JL, Ogilvie T. A review of thyroid cancer with intermediate differentiation. J Surg Oncol 2004;86:4454.

17. Lee YS, Kim Y, Jeon S, Bae JS, Jung SL, Jung CK. Cytologic, clinicopathologic, and molecular features of papillary thyroid carcinoma with prominent hobnail features: 10 case reports and systematic literature review. Int J Clin Exp Pathol 2015;8:7988-97.

18. Ieni A, Barresi V, Cardia R, Licata L, Di Bari F, Benvenga S, et al. The micropapillary/hobnail variant of papillary thyroid carcinoma: a review of series described in the literature compared to a series from one southern Italy pathology institution. Rev Endocr Metab Disord 2016;17:521-7.

19. Teng L, Deng W, Lu J, Zhang J, Ren X, Duan H, et al. Hobnail variant of papillary thyroid carcinoma: molecular profiling and comparison to classical papillary thyroid carcinoma, poorly differentiated thyroid carcinoma and anaplastic thyroid carcinoma. Oncotarget 2017;8:22023-33.

20. Ito M, Bogdanova T, Zurnadzhy L, Saenko V, Rogounovitch T, Mitsutake N, et al. Morphological difference in adult thyroid papillary carcinoma between Japan and Ukraine. En- 
docr J 2014;61:1221-8.

21. Sugitani I, Toda K, Yamamoto N, Sakamoto A, Fujimoto Y. Re-evaluation of histopathological factors affecting prognosis of differentiated thyroid carcinoma in an iodine-sufficient country. World J Surg 2010;34:1265-73.

22. Fukushima M, Ito Y, Hirokawa M, Akasu H, Shimizu K, Miyauchi A. Clinicopathologic characteristics and prognosis of diffuse sclerosing variant of papillary thyroid carcinoma in Japan: an 18-year experience at a single institution. World J Surg 2009;33:958-62.

23. Falvo L, Giacomelli L, D'Andrea V, Marzullo A, Guerriero G, de Antoni E. Prognostic importance of sclerosing variant in papillary thyroid carcinoma. Am Surg 2006;72:438-44.

24. Lam AK, Lo CY. Diffuse sclerosing variant of papillary carcinoma of the thyroid: a 35-year comparative study at a single institution. Ann Surg Oncol 2006;13:176-81.

25. Bai Y, Kakudo K, Li Y, Liu Z, Ozaki T, Ito Y, et al. Subclassification of non-solid-type papillary thyroid carcinoma identification of high-risk group in common type. Cancer Sci 2008;99:1908-15.

26. Nikiforov YE, Erickson LA, Nikiforova MN, Caudill CM, Lloyd RV. Solid variant of papillary thyroid carcinoma: incidence, clinical-pathologic characteristics, molecular analysis, and biologic behavior. Am J Surg Pathol 2001;25:147884.

27. DeLellis RA, Lloyd RV, Heitz PU, Eng C. Pathology and Genetics of Tumours of Endocrine Organs. 3rd ed. Lyon: IARC Press; 2004.

28. Ito Y, Hirokawa M, Miyauchi A, Higashiyama T, Kihara M, Miya A. Prognostic significance of the proportion of tall cell components in papillary thyroid carcinoma. World J Surg 2017;41:742-7.

29. Wang X, Cheng W, Liu C, Li J. Tall cell variant of papillary thyroid carcinoma: current evidence on clinicopathologic features and molecular biology. Oncotarget 2016;7:40792-9.

30. Lee SH, Jung CK, Bae JS, Jung SL, Choi YJ, Kang CS. Liquid-based cytology improves preoperative diagnostic accuracy of the tall cell variant of papillary thyroid carcinoma. Diagn Cytopathol 2014;42:11-7.

31. Kim SY, Kim T, Kim K, Bae JS, Kim JS, Jung CK. Highly prevalent BRAF V600E and low-frequency TERT promoter mutations underlie papillary thyroid carcinoma in Koreans.
J Pathol Transl Med 2020;54:310-7.

32. Shin JH. Ultrasonographic imaging of papillary thyroid carcinoma variants. Ultrasonography 2017;36:103-10.

33. Baek HJ, Kim DW, Shin GW, Heo YJ, Baek JW, Lee YJ, et al. Ultrasonographic features of papillary thyroid carcinomas according to their subtypes. Front Endocrinol (Lausanne) 2018;9:223.

34. Choi YJ, Shin JH, Kim JH, Jung SL, Son EJ, Oh YL. Tall cell variant of papillary thyroid carcinoma: sonographic and clinical findings. J Ultrasound Med 2011;30:853-8.

35. Ha EJ, Moon WJ, Na DG, Lee YH, Choi N, Kim SJ, et al. A multicenter prospective validation study for the Korean Thyroid Imaging Reporting and Data System in patients with thyroid nodules. Korean J Radiol 2016;17:811-21.

36. Yun MB, Sundar PS, Lan PY, Ying SX, Hua Z. Ultrasonographic features of diffuse sclerosing variant of papillary thyroid carcinoma. J Med Ultrasound 2011;19:41-6.

37. Chong Y, Shin JH, Oh YL, Han BK, Ko EY. Cribriformmorular variant of papillary thyroid carcinoma: ultrasonographic and clinical characteristics. Thyroid 2013;23:45-9.

38. Asioli S, Erickson LA, Sebo TJ, Zhang J, Jin L, Thompson GB, et al. Papillary thyroid carcinoma with prominent hobnail features: a new aggressive variant of moderately differentiated papillary carcinoma. A clinicopathologic, immunohistochemical, and molecular study of eight cases. Am J Surg Pathol 2010;34:44-52.

39. Vuong HG, Kondo T, Pham TQ, Oishi N, Mochizuki K, Nakazawa T, et al. Prognostic significance of diffuse sclerosing variant papillary thyroid carcinoma: a systematic review and meta-analysis. Eur J Endocrinol 2017;176:433-41.

40. Chen CC, Chen WC, Peng SL, Huang SM. Diffuse sclerosing variant of thyroid papillary carcinoma: diagnostic challenges occur with Hashimoto's thyroiditis. J Formos Med Assoc 2013;112:358-62.

41. Sheu SY, Schwertheim S, Worm K, Grabellus F, Schmid KW. Diffuse sclerosing variant of papillary thyroid carcinoma: lack of BRAF mutation but occurrence of RET/PTC rearrangements. Mod Pathol 2007;20:779-87.

42. Joung JY, Kim TH, Jeong DJ, Park SM, Cho YY, Jang HW, et al. Diffuse sclerosing variant of papillary thyroid carcinoma: major genetic alterations and prognostic implications. Histopathology 2016;69:45-53. 